\title{
The utilization of next-generation sequencing to detect somatic mutations and predict clinical prognosis of Chinese non-small cell lung cancer patients
}

This article was published in the following Dove Press journal: OncoTargets and Therapy

\author{
Liming Cao' \\ Long Long ${ }^{2}$ \\ Min $\mathrm{Li}^{1}$ \\ Huaping Yang' \\ Pengbo Deng' \\ Xinru $\mathrm{Mao}^{3}$ \\ Jianxing Xiang ${ }^{3}$ \\ Bing $\mathrm{Li}^{3}$ \\ Tengfei Zhang ${ }^{3}$ \\ Chengping $\mathrm{Hu}^{\prime}$ \\ 'Department of Respiratory Medicine, \\ Xiangya Hospital, Central South \\ University, Changsha, Hunan, China; \\ ${ }^{2}$ Department of Cardiothoracic \\ Surgery, Xiangya Hospital, Central \\ South University, Changsha, Hunan, \\ China; ${ }^{3}$ Burning Rock Biotech, \\ Guangzhou, China
}

Purpose: The development of next-generation sequencing (NGS) has revolutionized the understanding of oncogenesis of multiple types of cancer, including non-small cell lung cancer (NSCLC). However, there has been some debate over the utility of NGS for predicting patient prognosis and determining molecular targeted therapy. Therefore, we sought to demonstrate the numerous applications of NGS in the prognostic predictions and treatment of NSCLC patients.

Materials and methods: We performed NGS on either liquid or tissue tumor biopsies obtained from 53 NSCLC patients. The sequences were analyzed for oncogenic mutations, which were then correlated to clinical prognosis and smoking history.

Results: NGS of tumor biopsies detected both well-known driver mutations as well as rare or novel mutations. EGFR was the most frequently mutated gene, accounting for $32.4 \%$ (33/102) of the somatic mutations in this study. The EGFR mutations detected included rare variants such as EGFR exon 19 insertion (K745_E746insIPVAIK) and in cis H835L+L833V. Additionally, novel RET fusion mutations PCM1-RET and ADD3-RET were detected in two adenocarcinoma patients. To demonstrate the functional applications of NGS, we correlated mutations with patient characteristics, outcomes of matched targeted therapy, and outcomes based on allelic frequency of the EGFR-T790M mutation. Finally, we demonstrated that circulating tumor DNA can be used both to measure response to targeted therapy and as a predictor of clinical outcome, by presenting a case study of a single patient.

Conclusion: We demonstrated that NGS can be used in multiple applications to effectively identify potential oncogenic driver mutations, guide mutation-targeted therapy decisions, and predict clinical outcomes in Chinese NSCLC patients.

Keywords: next-generation sequencing, non-small cell lung cancer, prognosis

\section{Introduction}

Next-generation sequencing (NGS) has become a central technology used to detect somatic gene mutations in human cancer. ${ }^{1}$ NGS has multiple advantages over older, conventional genomic sequencing techniques. First, this high-throughput method can simultaneously detect numerous genetic variations over larger genomic regions. ${ }^{2}$ Second, NGS can be done accurately with a low input of DNA, which enables it to be used with a small amount of patient sample. Third, the high sensitivity and specificity makes NGS an effective and accurate tool for simultaneously identifying various mutation types, such as singlenucleotide variants, insertions and deletions, copy number variations, and gene fusions.
Correspondence: Chengping Hu Department of Respiratory Medicine, Xiangya Hospital, Central South University, 87 Xiangya Road, Changsha, Hunan 410018, China

$\mathrm{Tel}+86073 \mid 84328888$

$\mathrm{Fax}+86073184327332$

Email hcp282828@।26.com cc.
hereby accept the Terms. Non-commercial uses of the work are permitted without any further permission from Dove Medical Press Limited, provided the work is properly attributed. For permission for commercial use of this work, please see paragraphs 4.2 and 5 of our Terms (https://www.dovepress.com/terms.php). 
NGS may be able to revolutionize the treatment of lung cancer, which is a tumor type with a high mutational burden. ${ }^{3}$ Non-small cell lung cancer (NSCLC), one of the leading causes of cancer deaths worldwide, can be subdivided into adenocarcinoma, squamous cell carcinoma, and large cell carcinoma. ${ }^{4}$ Additionally, a subset of NSCLC patients harbor oncogenic mutations to the $E G F R$ gene, which subsequently promotes tumorigenesis and disease progression. ${ }^{5} E G F R$ transcribes the EGFR receptor tyrosine kinase protein, which activates multiple signaling pathways including cell proliferation, migration, and angiogenesis. ${ }^{6,7}$ The most frequent EGFR activating mutations in NSCLC are the deletion of exon 19 (19del) and L858R substitution. Together, these mutations occur in $10 \%-15 \%$ of western NSCLC patients and $40 \%$ of Asian NSCLC patients. ${ }^{8,9}$

Recently, targeted therapies have been developed to specifically inhibit cancer growth of tumors with certain mutations. ${ }^{10-12}$ For example, tumors with the EGFR $19 \mathrm{del}$ and L858R mutations displayed initial responses to first-generation tyrosine kinase inhibitors (TKIs) such as gefitinib and erlotinib. ${ }^{13}$ However, over $50 \%$ of patients experience disease progression after 9-14 months of treatment. This progression results from the development of the resistance-conferring EGFR T790M mutation. ${ }^{14-17}$ However, the third-generation EGFR-TKI osimertinib is a potent and irreversible EGFR inhibitor. Osimertinib effectively slows disease progression in NSCLC patients with both exon 19del and L858R mutations and T790M resistance mutations. The efficacy of osimertinib was first demonstrated in the AURA3 study, a Phase III clinical trial which investigated osimertinib vs platinum-based doublet chemotherapy in advanced NSCLC (ClinicalTrials.gov identifier: NCT02151981). Patients treated with osimertinib experienced a significantly longer median progression-free survival (PFS) than patients who received platinum therapy plus pemetrexed (10.1 vs 4.4 months).$^{18}$ However, the full potential of targeted therapies for treating patients with specific mutations is not fully appreciated in the clinical community due to limited clinical evidence demonstrating the ability of targeted therapy to improve patient outcomes.

In this study, we profiled tumor biopsies from 53 Chinese NSCLC patients by capture-based targeted ultra-deep sequencing. We identified driver mutations, rare mutations, and novel mutations in this cohort. Moreover, we demonstrated the ability of NGS to provide relevant clinical information to guide therapy. First, we correlated patient prognosis with the presence of specific somatic mutations. Second, we presented a case study to demonstrate that circulating tumor
DNA (ctDNA) value measured by NGS can be used to monitor and predict disease progression.

\section{Materials and methods}

\section{Patient selection}

This study recruited 53 patients with NSCLC and was approved by the Institutional Review Board at Xiangya Hospital. All patients provided written informed consent for this study.

\section{Tissue DNA and plasma cell-free DNA extraction}

Tissue DNA was extracted from biopsy samples using a QIAamp DNA FFPE tissue kit (Qiagen, Valencia, CA, USA) according to manufacturer's instructions. Circulating cell-free DNA was recovered from 4 to $5 \mathrm{~mL}$ of plasma using the QIAamp Circulating Nucleic Acid kit by Qiagen. After extraction, DNA concentration was quantified using a Qubit 2.0 Fluorometer (Thermo Fisher Scientific, Waltham, MA, USA).

\section{NGS library preparation and capture- based targeted DNA sequencing}

DNA shearing was performed using Covaris M220. End repair and A-tailing was followed by adaptor ligation. The ligated fragments with size of 200-400 bp were selected by beads (Agencourt AMPure XP Kit; Beckman Coulter, Brea, CA, USA), hybridized with probe baits, selected by magnetic beads, and amplified by polymerase chain reaction. Indexed samples were sequenced on a Nextseq500 sequencer (Illumina, Inc., San Diego, CA, USA), with paired-end reads.

\section{NGS data analysis pipeline}

All the reads were trimmed with Trimmomatic for adaptor and then mapped to the human genome (hg19) with the BurrowsWheeler Aligner. ${ }^{19,20}$ Local alignment optimization, mark duplication, and variant calling were performed using Genome Analysis ToolKit 3.2, ${ }^{21}$ Picard (http://picard.sourceforge.net), and VarScan. ${ }^{22}$ Gene rearrangements were called with Fusion And Chromosomal Translocation Enumeration and Recovery Algorithm (FACTERA), and copy number variation was analyzed with an in-house algorithm based on sequencing depth. ${ }^{23}$ Variants were filtered using the VarScan filter pipeline, with loci with depth $<100 \mathrm{X}$ filtered out. At least two supporting reads were needed for insertions and deletions in plasma samples and five supporting reads were needed for insertions and deletions in tissue samples. Single-nucleotide variants needed eight supporting reads to be called in both plasma 
and tissue samples. According to the ExAC, 1000 genomes, dbSNP, and ESP6500SI-V2 databases, variants with a population frequency over $0.1 \%$ were grouped as single-nucleotide polymorphisms and excluded from further analysis. Remaining variants were annotated with ANNOVAR and SnpEff v3.6. ${ }^{24,25}$

\section{Statistical analysis}

All data were analyzed using Software R. Kaplan-Meier analyses were performed to investigate the survival functions, and log-rank test was used to compare the difference between survival groups. Difference in EGFR frequency was calculated and presented using paired, two-tailed Student's $t$-test in $p$-value. For all statistical tests, $p<0.05$ was considered statistically significant.

\section{Results}

\section{Patient characteristics}

A total of 53 NSCLC patients were enrolled in this study. The baseline patient clinical characteristics are summarized in Table 1. Of the patients enrolled, 23 (43.4\%) were female and $30(56.6 \%)$ were male. The median age was 63 years (range: $36-82)$. Twenty-seven (50.9\%) patients had a history of smoking and 23 patients (43.4\%) were never smokers; information from 3 patients was lost. Among the histologic subtypes, 32 (60.4\%) patients were diagnosed with adenocarcinoma, 13 (24.5\%) with squamous cell carcinoma, and

Table I Summary of baseline patient characteristics

\begin{tabular}{|c|c|c|}
\hline Patient characteristics & $n=53$ & $\%$ \\
\hline \multicolumn{3}{|l|}{ Gender } \\
\hline Female & 23 & 43.4 \\
\hline Male & 30 & 56.6 \\
\hline \multicolumn{3}{|l|}{ Age, years } \\
\hline Mean & 62 & $\mathrm{~N} / \mathrm{A}$ \\
\hline Median & 63 & $\mathrm{~N} / \mathrm{A}$ \\
\hline Min & 36 & $\mathrm{~N} / \mathrm{A}$ \\
\hline Max & 82 & $\mathrm{~N} / \mathrm{A}$ \\
\hline \multicolumn{3}{|l|}{ Smoking history } \\
\hline Yes & 27 & 50.9 \\
\hline No & 23 & 43.4 \\
\hline Unknown & 3 & 5.7 \\
\hline \multicolumn{3}{|l|}{ Histologic types } \\
\hline LUAD & 32 & 60.4 \\
\hline LUSC & 13 & 24.5 \\
\hline LCLC & 1 & 1.9 \\
\hline Unknown & 7 & 13.2 \\
\hline \multicolumn{3}{|l|}{ Clinical stage } \\
\hline III & 3 & 5.7 \\
\hline IV & 33 & 62.3 \\
\hline Unknown & 17 & 32.1 \\
\hline
\end{tabular}

Abbreviations: LCLC, large cell lung cancer; LUAD, lung adenocarcinoma; LUSC lung squamous cell carcinoma; N/A, not applicable.
$1(1.9 \%)$ had large cell lung cancer; the other $7(13.2 \%)$ patients were also NSCLC patients, but without subtype records. Three $(5.7 \%)$ patients were diagnosed as stage III; $33(62.3 \%)$ patients were at stage IV.

\section{Mutation spectrum overview}

A total of 53 biopsies, consisting of 32 liquid samples and 21 tissue samples, were obtained from the 53 NSCLC patients. We performed capture-based targeted ultra-deep sequencing on these biopsies to identify somatic mutations by a wellestablished panel with high sensitivity and specificity. ${ }^{26}$ Corresponding white blood cells were used as a control to differentiate germline and somatic mutations. We achieved a mean average sequence depth of $12,628 \mathrm{X}$ for plasma samples and 1,214X for tissue samples.

We identified 102 somatic gene mutations in 35 (66\%) patients. The remaining 18 (34\%) patients had no somatic mutations detected. Of the mutations detected, there were 53 single-nucleotide variations, 17 insertions or deletions, 24 copy number amplifications, 3 fusions, 3 splice-site mutations, and 2 frame-shift mutations (Figure 1A). EGFR mutations were the most frequent, detected in 21 patients (39.6\%). Three other well-established oncogenic driver mutations were also detected among the 53 enrolled patients: KRAS G12X (3.8\%), RET rearrangement (1.9\%), and ERBB2 20ins (1.9\%), as shown in Figure 1B.

\section{NGS can be used to identify oncogenic driver mutations to direct drug selection for patients}

The specific EGFR mutation variant can be an independent prognostic factor and also direct a patient's therapy. To better understand which specific $E G F R$ mutation variants were present in patients, we analyzed the 33 detected $E G F R$ mutations detected in 21 patients. EGFR mutation subtypes identified in this cohort included exon 19del, T790M+exon 19del, L858R, T790M+L858R, exon 19ins (K745_E746insIPVAIK), H835L+L833V, and L861Q. K745_E746insIPVAIK was an uncommon insertion in exon 19 of $E G F R$, which was detected in an adenocarcinoma patient of our cohort (Figure 2A). ${ }^{27} \mathrm{We}$ detected another EGFR rare mutation, in cis $\mathrm{H} 835 \mathrm{~L}+\mathrm{L} 833 \mathrm{~V}$, which was located on the same allele in an adenocarcinoma patient (Figure 2B). Based upon the NGS result, the $\mathrm{H} 835 \mathrm{~L}+\mathrm{L} 833 \mathrm{~V}$ patient received gefitinib treatment and has achieved a PFS of 394 days till now. She had not experienced progressive disease (PD) and was continuing with gefitinib treatment with a good tolerance when this manuscript was submitted for publication. 


\section{A}

\section{Baseline samples}
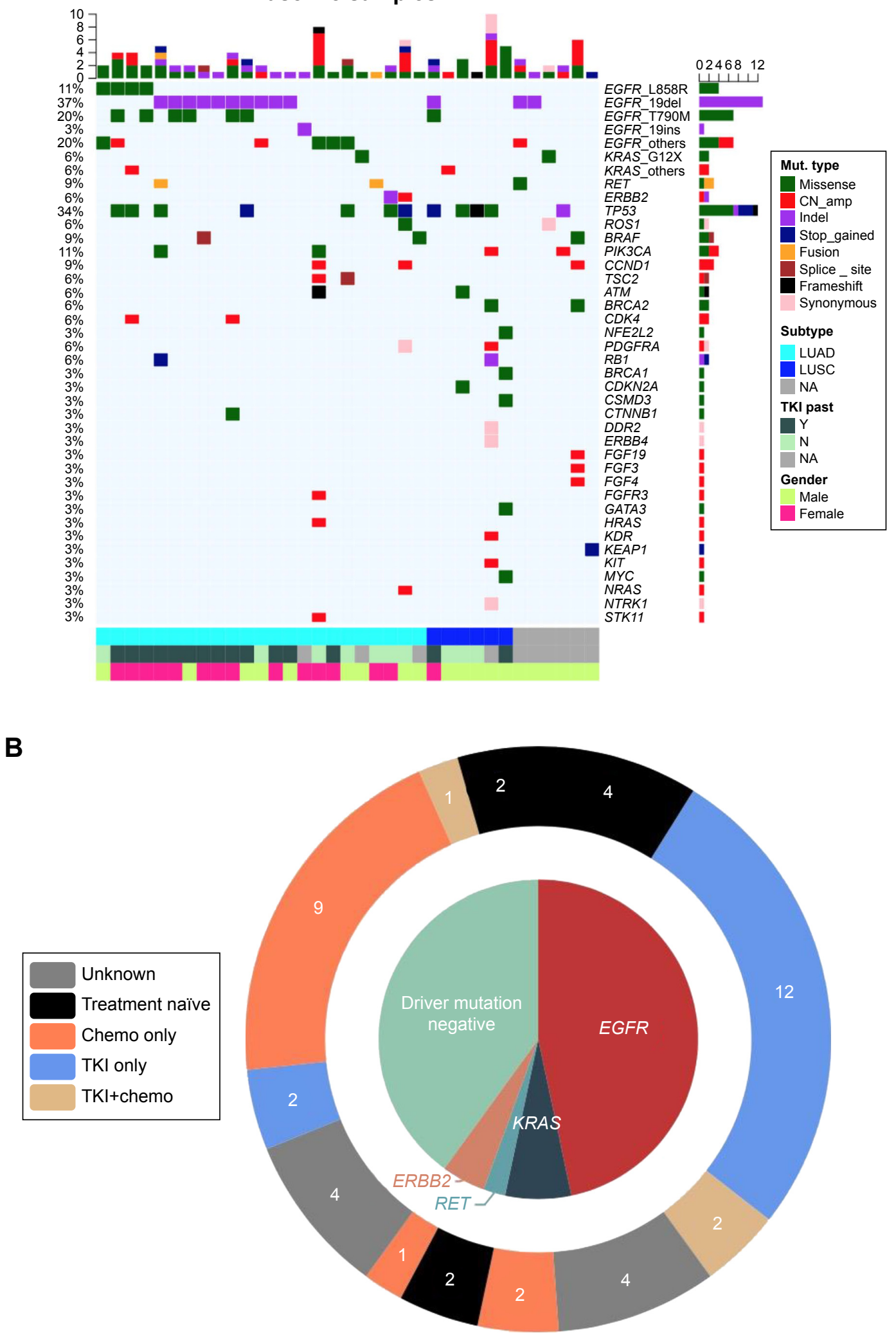

Figure I Baseline somatic gene aberrances identified by NGS.

Notes: (A) Mutation oncoprint identified at baseline of the 53 biopsies. Different colors show different types of baseline mutation. The top bar indicates the number of mutations identified in each individual patient; the side bar represents the number of patients having a certain mutation. Bottom categories with different colors indicate histologic subtypes, past TKI treatment history, and gender. (B) The schematic diagram demonstrates oncogenic driver mutations identified in our cohort. The outer ring displays the treatment history. The number in the outer ring indicates the number of patients who received corresponding past treatment.

Abbreviations: chemo, chemotherapy; CN_amp, copy number amplification; LUAD, lung adenocarcinoma; LUSC, lung squamous cell carcinoma; Mut, mutation; NA, not available; NGS, next-generation sequencing; TKI, tyrosine kinase inhibitor. 


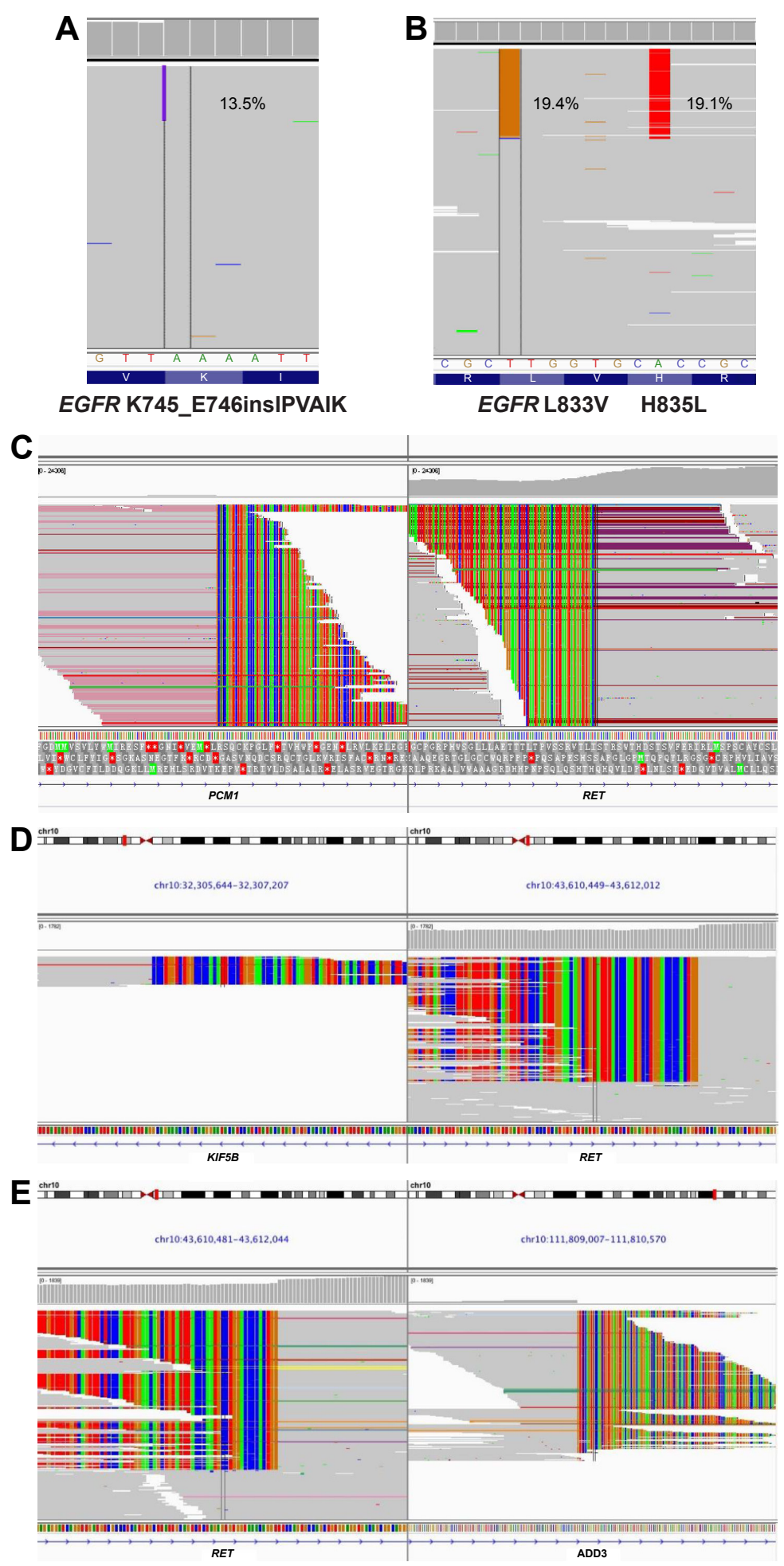

Figure 2 IGV screenshots displaying the reads of each mutation subtype identified by NGS.

Notes: (A) EGFR exon 19 insertion K745_E746insIPVAIK. (B) EGFR double-site mutation H835L+L833V located in cis. (C) PCMI-RET fusion. (D) KIF5B-RET fusion. (E) ADD3-RET fusion.

Abbreviations: IGV, Integrative Genomics Viewer; NGS, next-generation sequencing.

In addition to $E G F R$ mutations, we also identified two patients with three different variations of RET fusions, which are postulated to be an oncogenic mutations. First, a 40 -year-old advanced adenocarcinoma patient harbored both a PCM1-RET fusion and an EGFR exon 19del (Figure 2C). This is the first report of PCM1-RET fusion in lung adenocarcinoma, which has only been described in patients with papillary thyroid carcinoma. ${ }^{28}$ This patient 
received chemotherapy of cisplatin plus gemcitabine and achieved a PFS of 26 days before disease progression, suggesting that this fusion protein confers resistance to standard chemotherapy. We also identified a 60 -year-old female with advanced adenocarcinoma who harbored two different $R E T$ fusions - ADD3-RET and KIF5B-RET (Figure 2D and E). The $K I F 5 B-R E T$ fusion is an in-frame fusion of the KIF5B (the kinesin family $5 \mathrm{~B}$ gene) transcript and the RET oncogene. This fusion leads to abnormal activation of the $R E T$ kinase. It has previously been detected in $1 \%-2 \%$ of lung adenocarcinomas in both Japanese and American patients. ${ }^{29}$ In light of the recent reports of the KIF5B-RET fusion in lung adenocarcinoma, this mutation is postulated to be a driver mutation in these patients. The ADD3-RET fusion detected in this patient is an Intron2_Intron11 fusion, which has not been reported before. Therefore, further investigation is needed to validate the function of $A D D 3-R E T$ in lung adenocarcinoma oncogenesis.

\section{NGS validation of an increased frequency of EGFR mutations in NSCLC patients who have never smoked}

Previously, we reported that the EGFR sensitizing mutations occur more frequently in Chinese NSCLC patients without a history of smoking. ${ }^{30}$ We sought to validate these results with this study. Therefore, we collected detailed information of the clinical characteristics and baseline molecular alterations in 39 of the 53 patients sequenced in this study. We correlated the presence of EGFR sensitizing mutations and smoking history in this sub-cohort. Of the patients analyzed, EGFR sensitizing mutations were significantly more frequent in never smokers (51\%) than in smokers $(24 \%, p=0.023$; Figure $3 \mathrm{~A})$. The result further validated previous research demonstrating that the EGFR sensitizing mutations may drive oncogenesis in nonsmokers.

\section{Utilizing NGS to select mutation-targeted therapy and increase PFS}

Next, we sought to understand whether the utilization of NGS to treat patients with matched targeted therapy (MTT) correlated with patient prognosis. We collected patients who were either treated with MTT or not after the identification of driver mutation by NGS and performed Kaplan-Meier analysis to study the relationship of MTT and PFS. The result revealed that patients who received mutation-MTT achieved significantly longer median PFS than patients who did not (275 vs 80 days, $p<0.0001$; Figure 3B). Therefore, we concluded that NGS results should inform therapy decisions in patients with NSCLC when possible, as it significantly increases PFS.

Patients with EGFR activating mutations have excellent response to MTT with first-generation EGFR-TKIs. After treatment with these TKIs, patients frequently develop the EGFR T790M mutation, which can be targeted with the third-generation EGFR-TKI osimertinib. However, patient response to osimertinib is variable. ${ }^{31}$ To determine whether variation in allelic fraction of the T790M mutation could be a prognostic indicator of response to osimertinib, we analyzed the relationship of EGFR T790M allelic fraction and clinical outcomes. Patients harboring T790M mutation and receiving osimertinib treatment were divided into two groups: "allelic fraction high" and "allelic fraction low". The result showed that patients with a higher T790M allelic fraction had a shorter median PFS than patients with a lower T790M allelic fraction (238 vs 378 days, $p=0.059$; Figure $3 \mathrm{C}$ ). Therefore, the allelic fraction may be a prognostic indicator of response to osimertinib for patients with the T790M mutation.

\section{Utilization of serial liquid biopsies to predict disease progression}

Liquid biopsies of ctDNA can be used as a noninvasive method to monitor disease progression. Prior research suggests that using serial liquid biopsies in a patient after completion of chemotherapy can predict disease progression and help select targeted therapy based on the patient's mutational profile. ${ }^{32,33}$ Here, we performed longitudinal sequencing to further validate the ctDNA value for disease prediction.

To validate this technique, a single patient with stage IIIb squamous cell carcinoma who had experienced clinical PD after initial therapy with icotinib was selected. When enrolled in the study, the initial NGS analysis of his liquid biopsy revealed he had an EGFR L858R substitution and $K R A S$ amplification. He was next treated with a combination of pemetrexed, carboplatin, and bevacizumab, which resulted in clinically stable disease (SD). Exactly 122 days after therapy, a liquid biopsy was taken for NGS. Results of NGS demonstrated a significant reduction of total ctDNA, which confirmed his SD. Additionally, the patient had a significant decrease of the allelic fraction of the EGFR L858R substitution and the KRAS amplification previously detected. Six months after initiation of therapy, the patient underwent a third liquid biopsy. At this time, ctDNA sequencing revealed an increased allelic frequency of $E G F R$ L858R, KRAS amplification, and ctDNA. This ctDNA change preceded the 


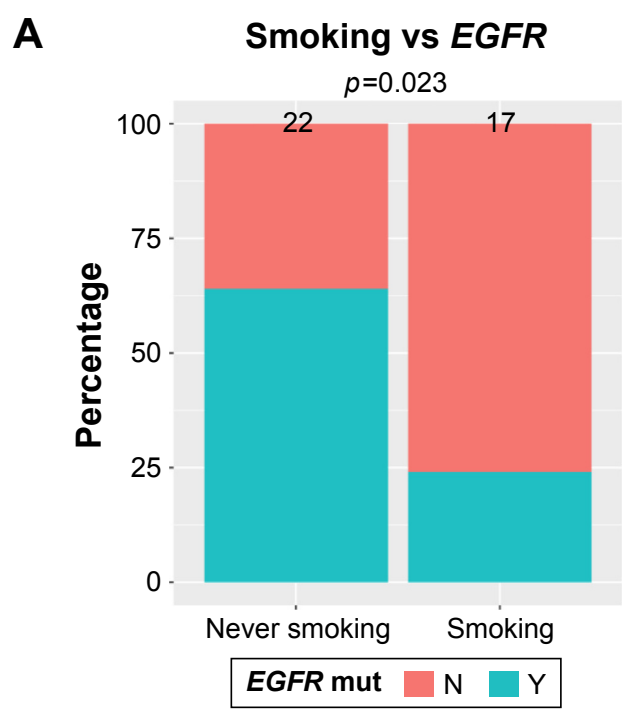

B

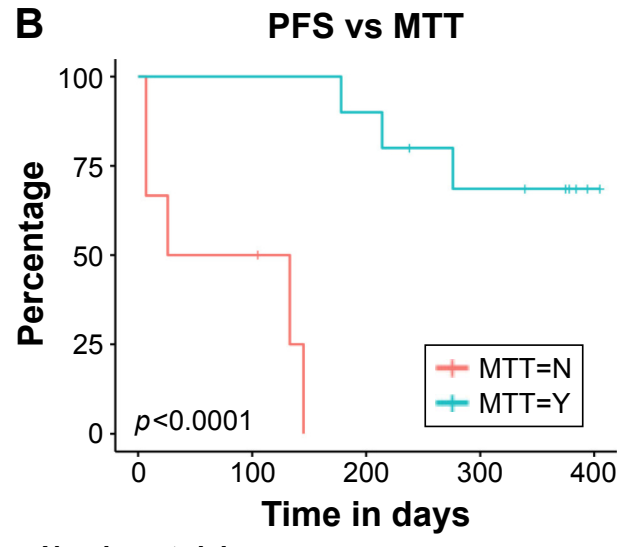

Number at risk

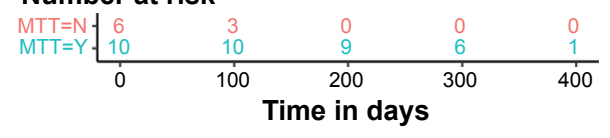

C PFS vs AF of T790M

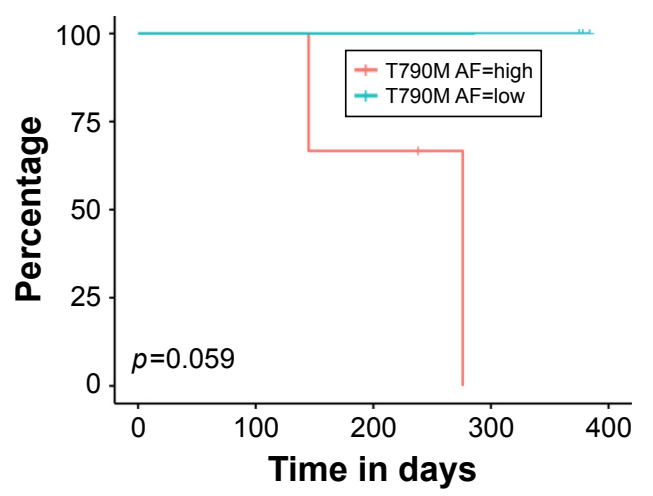

Number at risk

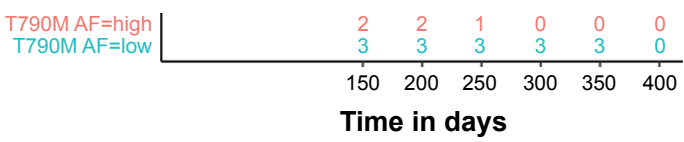

Figure 3 Correlation of patient baseline characteristics, baseline somatic aberrances, and PFS.

Notes: (A) The correlation of EGFR mutation occurrence frequency and smoking status. (B) Patients who received MTT commonly achieved longer PFS. (C) Allelic fraction of EGFR T790M was inversely correlated with PFS. Difference of EGFR mutation percentage between subgroups (never smoker vs smoker) was calculated with Student's $t$-test; a two-sided $p$-value of $<0.05$ was considered statistically significant. A Kaplan-Meier analysis was used to estimate survival, and a log-rank test was used to determine the difference in survival curve between subgroups.

Abbreviations: AF, allelic fraction; MTT, matched targeted therapy; PFS, progression-free survival.

detection of clinical PD revealed by radiographic imaging by 46 days (Figure 4$)$.

\section{Discussion}

In this study, we performed capture-based targeted ultra-deep sequencing on 53 biopsies from Chinese NSCLC patients. Our results demonstrated that molecular profiling of biopsies from cancer patients can be used to stratify prognosis outcomes and predict disease progression in patients.

Using NGS, we identified several rare mutations in this lung cancer cohort. Here, we reported the first case of $P C M 1-R E T$ fusion in adenocarcinoma, which was previously only discovered in papillary thyroid carcinoma. We also identified a patient with a triple fusion of $A D D 3-R E T$, which has never been described in any cancer subtype. Previous research has demonstrated that RET fusions promote oncogenesis both in solid tumors and in hematologic malignancies. ${ }^{34}$ Additionally, the patient with the ADD3-RET mutation experienced poor outcomes to therapy, suggesting it may be an indicator of poor prognosis. Future research investigating the oncogenic functions of these two RET fusions will be important to understand their role in disease progression and provide basic knowledge for developing targeted therapies to these fusions. 


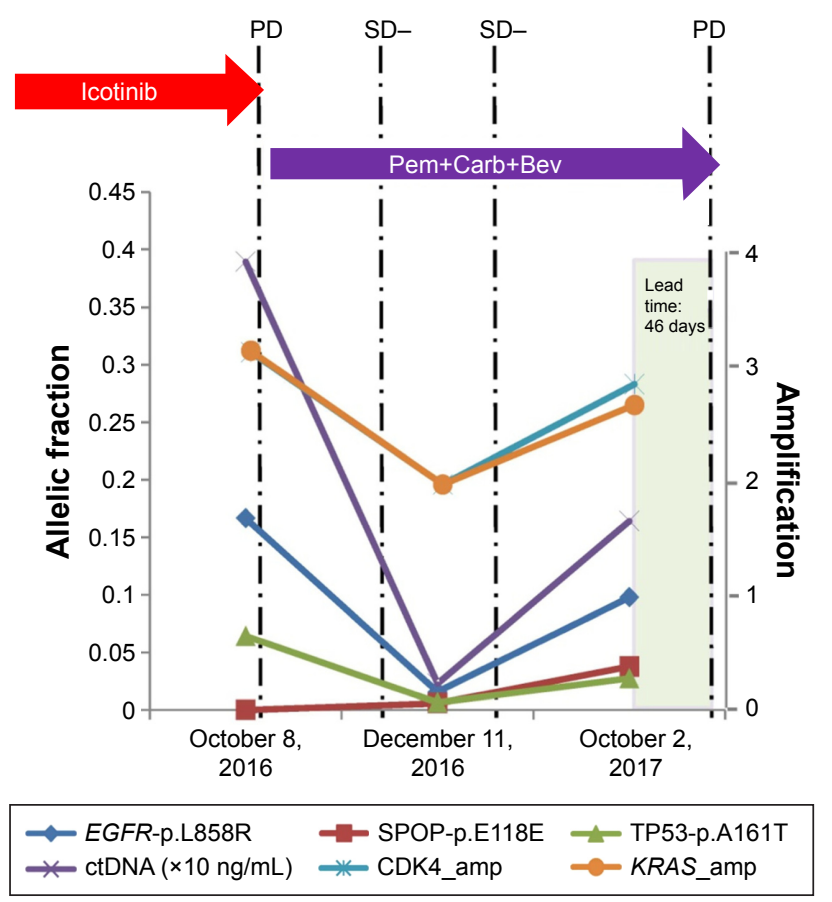

Figure $4 \mathrm{~A}$ lung squamous cell carcinoma patient is presented here to illustrate the value of ctDNA in predicting disease progression earlier than radiographic imaging. Notes: With the development of EGFR L858R and KRAS amplification, which leads to icotinib resistance, he was treated with Pem+Carb+Bev therapy and achieved clinical SD. Afterwards, sequencing of liquid biopsy revealed the increased allelic fraction of EGFR L858R, KRAS amplification, and ctDNA level, which were earlier than clinical PD detected by radiographic imaging for a period of 46 days. This case demonstrated the value of ctDNA for early detection of disease progression than radiological PD.

Abbreviations: amp, amplification; ctDNA, circulating tumor DNA; PD, progressive disease; Pem+Carb+Bev, pemetrexed+carboplatin+bevacizumab; SD, stable disease.

In addition to $R E T$ fusions, we also identified an insertion mutation to EGFR exon 19. In NSCLC, this is a rare event which only occurs in $1 \%-2 \%$ of patients. In this study, a single adenocarcinoma patient harbored an EGFR exon 19ins (K745_E746insIPVAIK). Although exon 19 insertions are rare, this K745_E746insIPVAIK comprises $41.1 \%$ of insertion mutations in EGFR exon 19. ${ }^{35,36}$ Molecular models of the EGFR K745_E746insIPVAIK suggest that the mutation modifies the kinase domain. This may facilitate the binding of TKIs, leading to sensitivity to EGFR-TKI. ${ }^{36}$ Although therapy decisions for this patient were not available, the presence of this mutation would suggest he would benefit from targeted therapy with a TKI.

In this cohort of patients, we also identified an in cis $\mathrm{H} 835 \mathrm{~L}+\mathrm{L} 833 \mathrm{~V}$ dual substitution mutation in an adenocarcinoma patient. The presence of both these mutations in a single patient is consistent with previous publications reporting that $\mathrm{H} 835 \mathrm{~L}$ mutations are frequently combined with $\mathrm{L} 833 \mathrm{~V}$ mutation in cis. $^{37-39}$ In our study, this patient responded well to therapy with the TKI gefitinib. The response was maintained for 394 days, and the patient had not progressed at the time of submission of this manuscript. This corroborates with case reports from two other groups. Frega et al presented a lung adenocarcinoma patient harboring EGFR in cis H835L+L833V who achieved a PR to afatinib. ${ }^{39}$ Yang et al also reported that a lung adenocarcinoma patient with in cis $\mathrm{H} 835 \mathrm{~L}+\mathrm{L} 833 \mathrm{~V}$ achieved a near complete response which was maintained for 34 weeks after gefitinib treatment. ${ }^{38}$

In addition to the identification of rare genetic mutations, we correlated patient prognosis with baseline oncogenic mutations. Previously, an analysis of Japanese lung cancer patients who underwent potentially curative pulmonary resection reported that EGFR mutations occur more frequently in patients with no history of smoking $(66 \%)$ than in those patients who smoked (22\%). ${ }^{30}$ In our study, Chinese NSCLC patients with diverse treatment histories confirmed the conclusion that $E G F R$ mutations occur more frequently in patients who had never smoked ( $p=0.023$ ). One possible assumption is that $E G F R$ mutation might be caused by carcinogens other than those contained in tobacco.

The EGFR T790M mutation accounts for $>50 \%$ of acquired TKI resistance and can be overcome by the EGFRTKI osimertinib. Oxnard et al reported that NSCLC patients with the T790M mutation had better outcomes to osimertinib (overall response rate [ORR], 69\%; PFS=16.5 months) than T790M-negative patients (ORR, 25\%; PFS $=2.8$ months). ${ }^{40}$ Our study demonstrated not only the presence of T790M mutation, but also the allelic frequency correlated with patient outcomes. In this cohort of patients, the EGFR T790M allelic frequency was inversely associated with PFS.

The development of mutation-targeted therapies, coupled with NGS, suggests that a patient's molecular profile can be used to guide therapy. However, the clinical benefit of targeted therapy vs conventional therapy remains a controversy. A small study of patients who underwent targeted therapy reported that $27 \%$ of cancer patients achieved a $30 \%$ increase in PFS after targeted therapy, as compared to their previous, nontargeted treatment. ${ }^{41}$ Similarly, a retrospective study demonstrated that patients treated with targeted therapy demonstrated improved PFS and overall survival. ${ }^{42}$ On the other hand, a French study carried out in 741 cancer patients reported that no significant difference occurred between patients who received molecular targeted therapy compared with those receiving conventional treatment. ${ }^{43}$ In our study, we demonstrated that patients receiving matchtargeted therapy achieved a significantly longer median PFS than patients who did not. Potentially, the heterogeneity of oncogenic driver mutations in different cohorts can lead to different responses to targeted drugs. Therefore, further 
prospective investigation of the benefits of targeted therapy in specific patient populations can address the challenge of patient heterogeneity and identify the most representative patients for targeted therapy. Plasma ctDNA can be used not only to estimate tumor burden at diagnosis, but also as a predictor of tumor progression by dynamically monitoring the clonal changes. ${ }^{44,45}$ Previously, a study of patients with stage II colon cancer demonstrated that the presence of ctDNA after operation was strongly correlated with disease recurrence. ${ }^{32}$ An increase in ctDNA has also been demonstrated to be an earlier predictor of clinical response and disease progression than other biomarkers, such as CA $15-3 .{ }^{33}$ In this study, we presented a case study demonstrating the value of dynamically monitoring ctDNA to predict disease progression. The increase in levels of ctDNA occurred much earlier than clinical PD by radiographic imaging. It highlights the notion that plasma ctDNA is a noninvasive tool for disease monitoring and PD early detection.

In conclusion, we demonstrated that NGS has numerous applications which can be used to understand the molecular characteristics of NSCLC. The ever-decreasing costs of applying the technology in the clinic make it a realistic tool that physicians can utilize to identify mutations which may guide therapy decisions. Future studies to prospectively demonstrate the application of NGS in both NSCLC and other tumors are needed to confirm the benefit of this tool to cancer treatment.

\section{Disclosure}

The authors report no conflicts of interest in this work.

\section{References}

1. Gagan J, Van Allen EM. Next-generation sequencing to guide cancer therapy. Genome Med. 2015;7(1):80.

2. Luthra R, Chen H, Roy-Chowdhuri S, Singh RR. Next-generation sequencing in clinical molecular diagnostics of cancer: advantages and challenges. Cancers (Basel). 2015;7(4):2023-2036.

3. Chalmers ZR, Connelly CF, Fabrizio D, et al. Analysis of 100,000 human cancer genomes reveals the landscape of tumor mutational burden. Genome Med. 2017;9(1):34.

4. Travis WD, Brambilla E, Burke AP, Marx A, Nicholson AG. Introduction to the 2015 World Health Organization classification of tumors of the lung, pleura, thymus, and heart. J Thorac Oncol. 2015;10(9): 1240-1242.

5. Ji H, Li D, Chen L, et al. The impact of human EGFR kinase domain mutations on lung tumorigenesis and in vivo sensitivity to EGFR-targeted therapies. Cancer Cell. 2006;9(6):485-495.

6. Arteaga CL. Overview of epidermal growth factor receptor biology and its role as a therapeutic target in human neoplasia. Semin Oncol. 2002;29(5 Suppl 14):3-9.

7. Bunn PA Jr, Franklin W. Epidermal growth factor receptor expression, signal pathway, and inhibitors in non-small cell lung cancer. Semin Oncol. 2002;29(5 Suppl 14):38-44.
8. Pao W, Chmielecki J. Rational, biologically based treatment of EGFR-mutant non-small-cell lung cancer. Nat Rev Cancer. 2010; 10(11):760-774.

9. Xia N, An J, Jiang QQ, Li M, Tan J, Hu CP. Analysis of EGFR, EML4ALK, KRAS, and c-MET mutations in Chinese lung adenocarcinoma patients. Exp Lung Res. 2013;39(8):328-335.

10. Wang LY, Cui JJ, Guo AX, Yin JY. Clinical efficacy and safety of afatinib in the treatment of non-small-cell lung cancer in Chinese patients. Onco Targets Ther. 2018;11:529-538.

11. Qu J, Wang YN, Xu P, et al. Clinical efficacy of icotinib in lung cancer patients with different EGFR mutation status: a meta-analysis. Oncotarget. 2017;8(20):33961-33971.

12. Ge L, Shi R. Progress of EGFR-TKI and ALK/ROS1 inhibitors in advanced non-small cell lung cancer. Int J Clin Exp Med. 2015;8(7): 10330-10339.

13. Lu RL, Hu CP, Yang HP, Li YY, Gu QH, Wu L. Biological characteristics and epidermal growth factor receptor tyrosine kinase inhibitors efficacy of EGFR mutation and its subtypes in lung adenocarcinoma. Pathol Oncol Res. 2014;20(2):445-451.

14. Maemondo M, Inoue A, Kobayashi K, et al. Gefitinib or chemotherapy for non-small-cell lung cancer with mutated EGFR. $N$ Engl J Med. 2010;362(25):2380-2388.

15. Mitsudomi T, Morita S, Yatabe Y, et al. Gefitinib versus cisplatin plus docetaxel in patients with non-small-cell lung cancer harbouring mutations of the epidermal growth factor receptor (WJTOG3405): an open label, randomised phase 3 trial. Lancet Oncol. 2010;11(2):121-128.

16. Mok TS, Wu YL, Thongprasert S, et al. Gefitinib or carboplatinpaclitaxel in pulmonary adenocarcinoma. NEngl J Med. 2009;361(10): 947-957.

17. Rosell R, Carcereny E, Gervais R, et al. Erlotinib versus standard chemotherapy as first-line treatment for European patients with advanced EGFR mutation-positive non-small-cell lung cancer (EURTAC): a multicentre, open-label, randomised phase 3 trial. Lancet Oncol. 2012;13(3): 239-246.

18. Mok TS, Wu YL, Ahn MJ, et al. Osimertinib or platinum-pemetrexed in EGFR T790M-positive lung cancer. $N$ Engl J Med. 2017; 376(7):629-640.

19. Bolger AM, Lohse M, Usadel B. Trimmomatic: a flexible trimmer for Illumina sequence data. Bioinformatics. 2014;30(15):2114-2120.

20. Li H, Durbin R. Fast and accurate short read alignment with BurrowsWheeler transform. Bioinformatics. 2009;25(14):1754-1760.

21. McKenna A, Hanna M, Banks E, et al. The Genome Analysis Toolkit: a MapReduce framework for analyzing next-generation DNA sequencing data. Genome Res. 2010;20(9):1297-1303.

22. Koboldt DC, Zhang Q, Larson DE, et al. VarScan 2: somatic mutation and copy number alteration discovery in cancer by exome sequencing. Genome Res. 2012;22(3):568-576.

23. Newman AM, Bratman SV, Stehr H, et al. FACTERA: a practical method for the discovery of genomic rearrangements at breakpoint resolution. Bioinformatics. 2014;30(23):3390-3393.

24. Wang K, Li M, Hakonarson H. ANNOVAR: functional annotation of genetic variants from high-throughput sequencing data. Nucleic Acids Res. 2010;38(16):e164.

25. Cingolani P, Platts A, Wang le L, et al. A program for annotating and predicting the effects of single nucleotide polymorphisms, SnpEff: SNPs in the genome of Drosophila melanogaster strain w1118; iso-2; iso-3. Fly (Austin). 2012;6(2):80-92.

26. Mao X, Zhang Z, Zheng X, et al. Capture-based targeted ultradeep sequencing in paired tissue and plasma samples demonstrates differential subclonal ctDNA-releasing capability in advanced lung cancer. J Thorac Oncol. 2017;12(4):663-672.

27. Agbarya A, Melamed-Frank M, Kaidar-Person O, et al. Getting out of a wheelchair: an uncommon insertion mutation in exon 19 of EGFR responsive to erlotinib. Springerplus. 2014;3:507.

28. Corvi R, Berger N, Balczon R, Romeo G. RET/PCM-1: a novel fusion gene in papillary thyroid carcinoma. Oncogene. 2000;19(37): 4236-4242. 
29. Kohno T, Ichikawa H, Totoki Y, et al. KIF5B-RET fusions in lung adenocarcinoma. Nat Med. 2012;18(3):375-377.

30. Kosaka T, Yatabe Y, Endoh H, Kuwano H, Takahashi T, Mitsudomi T. Mutations of the epidermal growth factor receptor gene in lung cancer: biological and clinical implications. Cancer Res. 2004;64(24):8919-8923.

31. Yang JC, Ahn MJ, Kim DW, et al. Osimertinib in Pretreated T790MPositive Advanced Non-Small-Cell Lung Cancer: AURA Study Phase II Extension Component. J Clin Oncol. 2017;35(12):1288-1296.

32. Tie J, Wang Y, Tomasetti C, et al. Circulating tumor DNA analysis detects minimal residual disease and predicts recurrence in patients with stage II colon cancer. Sci Transl Med. 2016;8(346):346ra392.

33. Dawson SJ, Tsui DW, Murtaza M, et al. Analysis of circulating tumor DNA to monitor metastatic breast cancer. N Engl J Med. 2013; 368(13):1199-1209.

34. Ferrara R, Auger N, Auclin E, Besse B. Clinical and translational implications of RET rearrangements in non-small cell lung cancer. J Thorac Oncol. 2018;13(1):27-45.

35. He M, Capelletti M, Nafa K, et al. EGFR exon 19 insertions: a new family of sensitizing EGFR mutations in lung adenocarcinoma. Clin Cancer Res. 2012;18(6):1790-1797.

36. Otto C, Csanadi A, Fisch P, Werner M, Kayser G. Molecular modeling and description of a newly characterized activating mutation of the EGFR gene in non-small cell lung cancer. Diagn Pathol. 2012;7:146.

37. Zhuang Y, Xu J, Ma H, et al. A sequential method of epidermal growth factor receptor mutation detection reduces false negatives: a new case with doublet mutations of L833V and H835L in China. Clin Lung Cancer. 2013;14(3):295-300.

38. Yang TY, Tsai CR, Chen KC, Hsu KH, Lee HM, Chang GC. Good response to gefitinib in a lung adenocarcinoma harboring a heterozygous complex mutation of $\mathrm{L} 833 \mathrm{~V}$ and $\mathrm{H} 835 \mathrm{~L}$ in epidermal growth factor receptor gene. J Clin Oncol. 2011;29(16):e468-e469.
39. Frega S, Conte P, Fassan M, Polo V, Pasello G. A Triple rare E709K and L833V/H835L EGFR mutation responsive to an irreversible pan-HER inhibitor: a case report of lung adenocarcinoma treated with afatinib. J Thorac Oncol. 2016;11(5):e63-e64.

40. Oxnard GR, Thress KS, Alden RS, et al. Association between plasma genotyping and outcomes of treatment with osimertinib (AZD9291) in advanced non-small-cell lung cancer. J Clin Oncol. 2016;34(28): 3375-3382.

41. Von Hoff DD, Stephenson JJ Jr, Rosen P, et al. Pilot study using molecular profiling of patients' tumors to find potential targets and select treatments for their refractory cancers. J Clin Oncol. 2010;28(33): 4877-4883.

42. Tsimberidou AM, Iskander NG, Hong DS, et al. Personalized medicine in a phase I clinical trials program: the MD Anderson Cancer Center initiative. Clin Cancer Res. 2012;18(22):6373-6383.

43. Le Tourneau C, Delord JP, Goncalves A, et al. Molecularly targeted therapy based on tumour molecular profiling versus conventional therapy for advanced cancer (SHIVA): a multicentre, open-label, proof-of-concept, randomised, controlled phase 2 trial. Lancet Oncol. 2015;16(13): 1324-1334

44. Pecuchet N, Zonta E, Didelot A, et al. Base-position error rate analysis of next-generation sequencing applied to circulating tumor DNA in non-small cell lung cancer: a prospective study. PLoS Med. 2016; 13(12):e1002199.

45. Wan JCM, Massie C, Garcia-Corbacho J, et al. Liquid biopsies come of age: towards implementation of circulating tumour DNA. Nat Rev Cancer. 2017;17(4):223-238.
OncoTargets and Therapy

\section{Publish your work in this journal}

OncoTargets and Therapy is an international, peer-reviewed, open access journal focusing on the pathological basis of all cancers, potential targets for therapy and treatment protocols employed to improve the management of cancer patients. The journal also focuses on the impact of management programs and new therapeutic agents and protocols on

\section{Dovepress}

patient perspectives such as quality of life, adherence and satisfaction. The manuscript management system is completely online and includes a very quick and fair peer-review system, which is all easy to use. Visit http://www.dovepress.com/testimonials.php to read real quotes from published authors. 